\title{
Evolutionary Tuning Method for Automatic Impedance Matching in Communication Systems
}

\author{
Yichuang Sun and Wai Kit Lau \\ Department of Electrical and Electronic Engineering, Faculty of Engineering and Information Sciences \\ University of Hertfordshire, Hatfield, Herts AL10 9AB, UK \\ Tel: 441707 284196, Fax: 441707 284199, Email: y.sun@herts.ac.uk
}

\begin{abstract}
An evolutionary tuning method for automatic impedance matching in radio communication systems is developed based on Genetic Algorithms (GA). A detailed tuning procedure is presented and a software package has been developed. Simulation results using the software package are given. The implementation of the proposed tuning algorithm in a microprocessor-controlled automatic antenna tuning unit is also considered.
\end{abstract}

\section{Introduction}

Impedance matching networks and antenna tuning units have been used to achieve an optimum coupling for signals being transmitted or received through tuning of their components for a conjugate match between the transmitter or receiver impedance and antenna impedance [1-8]. The electromechanical tuning method usually has poor reliability and maintainability, high volume and weight, and inefficient detection and direction finding. It also takes a long tuning time to obtain an impedance match. Thus automatic tuning based on microcomputer control has been investigated. Four methods have been proposed [1]: the linear method, the binary-weight method, the phase difference nulling method and the subband tuning method. The linear method requires a large number of tuning steps for a small increment value and it is not possible to achieved the desired VSWR for large increment values. The weight method uses a binary switching technique which is convenient for control, but still requires a step-by-step and element-by-element tuning implementation. The phase difference nulling method is due to have matching problems at frequencies below $4 \mathrm{MHz}$. The subband tuning is antenna specific and reprogramming of the non-volatile EPROM or RAM has to be done whenever a different type of antenna is coupled to the matching network. These methods are thus either slow and not optimum or have limitations to the frequencies and antennas. Some random global research and general tuning method is therefore required to overcome the above problems [7]. Note in particular that for a network there may exist a large number of component values that can match a pair of impedances, especially when the network is complex $[5,6]$. Since any set of values will satisfy impedance matching, the random global search method may be generally faster that the regular step-by-step search approach for a solution of matching. The optimum and fast tuning strategies and algorithms may therefore be developed based on random global search methods.

Genetic Algorithms (GA) $[9,10]$ are random global search algorithms. They are based on the idea of natural selection, a biological process in which the fitter individuals will survive in the competing environment. The population which is formed using bits and pieces of the individuals will improve from generation to generation until a population is arrived at, containing one or more good solutions. The whole idea is that the fittest of the generations will survive. Since it was first developed, Genetic Algorithms has been widely used for problem solving in many areas. The availability of highspeed microcomputers has greatly encouraged its application in engineering areas since fast computation of these algorithms is no longer a major problem.

These are attractive features of the Genetic Algorithms that have encouraged its application in the fast and optimum tuning of impedance matching networks and antenna tuning units. It works with a binary encoded parameter set, uses cost function values in the optimization (not function derivatives), searches globally for a solution and exploits the accumulated information during evolution of the search. This paper investigates how to use Genetic Algorithms to achieve a fast and optimum tuning method for automatic impedance matching in communication systems.

\section{GA-based Tuning Method}

A tuning method based on GA will generate the component values that are required to produce a conjugate match between a particular pair of impedances for operation frequencies within a prescribed accuracy in a evolutionary way. This may take as few as several generations. The tuning algorithm is implemented in the control unit in the antenna tuning system, as shown in figure 1. The control unit will process the impedance and frequency information obtained from the monitoring or measurement unit and tune the components in the impedance matching network to match the transmitter/receiver impedance and antenna impedance.

For the matching network its component value ranges, $C_{j \max }, C_{j \min }, L_{j \max }$ and $L_{j \min }$ can be determined for the impedances to be matched and the frequencies of operation [6]. Conversely, if the component value ranges are known we can also determine the antenna 
impedance region that can be conjugate matched [5]. The tuning algorithm will work with binary discrete elements in the matching network. Each component in the network will be formed by a binary array of elements with switches as shown in figure 2 . The lowest element value will be the component minimum value, while the maximum component value can be achieved when all switches are closed. The idea behind this arrangement can be expressed as

$C_{1}=\left(a_{0} 2^{0}+a_{1} 2^{1}+\ldots+a_{n 1} 2^{n 1}\right) C_{1 \text { min }}$,

$\mathrm{L}=\left(\mathrm{b}_{0} 2^{0}+\mathrm{b}_{1} 2^{1}+\ldots+\mathrm{b}_{n} 2^{\mathrm{n}}\right) \mathrm{L}_{\text {min }}$

The coefficients are either 1 or 0 that represents the switch condition. Therefore the component values can be changed by physically switching the switches. Take $C_{1}$ as an example. There are $2^{\text {nl }}$ different values for $C_{1}$. The number of elements within each component array can be determined by using the equation below.

$\mathrm{nl}=\log _{2}\left(1+\mathrm{C}_{1 \max } / \mathrm{C}_{1 \min }\right)$

For the computation purpose, a string of coefficients in the form of binary values will represent each decimal component value. These strings of binary values for all tunable components will be combined into a single string called a "Chromosome" or an "Individual ". A number of chromosomes will then be used in the GAbased evolutionary tuning process.

Several equivalent specifications can be used to reflect the conjugate match or the maximum power transfer. This embraces the impedances, power, reflection coefficient, and voltage standing wave ratio (VSWR). There have been many methods which can be used to measure these parameters in antenna tuning units. In the development of the GA-based tuning algorithm, the cost function that is used to evaluate the fitness of each set of parameters or chromosomes is constructed based on the VSWR. The VSWR is most widely used to indicate whether a conjugate match has been achieved in antenna matching circuits. Ideally, the VSWR will have a value of 1 when a conjugate match is achieved. However, in practice, the VSWR that falls in between 1 and 1.5 may be considered acceptable $[1-4,7,8]$.

The VSWR, denoted by $\sigma$, can be obtained by

$\sigma=\frac{1+|\Gamma|}{1-|\Gamma|}$

where $\Gamma$ is the complex reflection coefficient, which can be calculated in terms of the input impedance, $Z_{\text {in, }}$ and the source resistance, $R_{1}$, given by

$\Gamma=\frac{Z_{\text {in }}-R_{1}}{Z_{\text {in }}+R_{1}}$

It can be shown that there may be a wide range of component values that can match $Z_{\text {in }}$ and $R_{1}$. In practice, a perfect impedance match is impossible due to various issues such as parasitic effects $[7,8]$. As a result the $\sigma=1, Z_{\text {in }}=R_{1}$ and $\Gamma=0$ conditions can never be achieved. In most cases, a maximum allowed value, $\sigma_{\max }$ is prescribed and the network should be tuned to the extent that $\sigma \leq \sigma_{\max }$.

We now summarize the GA-based evolutionary matching algorithm as below:

1). The first step is to obtain the information that is essential to perform the tuning process. The impedances to be matched and frequencies of operation, the ranges of component values (the number of binary elements in each component or the "Population" size) are required. Information signaling the stop of the tuning process such as the required value of the VSWR or the prescribed number of generations are also needed.

2). Once all the information is obtained, the tuning algorithm will first generate the initial pool of parameter sets which are also called the initial population. The parameters in each set are randomly generated within the respective given component ranges.

3 ). The following coding process will code each set of parameters into binary strings of coefficients based on the discrete binary element technique that is discussed previously. Each string of coefficients represents the binary coding of a parameter. These strings will then be combined to produce a single string of coefficients called the "Chromosome" which will represent a single set of parameters. A number of such chromosomes will form a population.

4). The fitness of each produced chromosome string will be evaluated using the VSWR, which was discussed before. The best fit chromosome or "Citizen" in that particular population will be determined.

5). After the initial population generation is completed, the Genetic Algorithms procedure will commence. The Genetic Algorithms consist of the following processes: Parent selection, Crossover, Mutation and Reinsertion They are performed in the order as shown. In the parent selection process, the choice of selection is based on the "Tournament Selection". It chooses two citizens at random, and the one with a better fitness gets to be the "Parent". This is a standard selection technique. As for the crossover, the "Uniform Crossover" is performed. It is the most commonly used technique where for each binary value in the "Child Chromosome", it is randomly chosen from one of the selected parents. The "Creep and Jump" mutations are performed in the mutation process. The creep mutation is done by randomly switching the binary value in a chromosome, while the jump mutation will randomly swap two adjacent bits in the same chromosome. Finally, for the reinsertion process, the best-fit individual of the previous generation or the best-fit parent will replace the weakest individual in the current generation or the weakest "Child".

6). The processes in Step 5 will be repeated until the generation loop has reached the required number of generations or the specified value of the VSWR. During 
each generation, a set of parameter values with the best fitness will be shown and a graph will also be plotted using this information.

\section{Simulation Results and Comments}

A general software package implementing the whole tuning algorithm described above has been developed in the $\mathrm{C}$.language. It has been tested. using practical examples. Simulation results for the $\pi$ network in figure 3 are now presented ( the $\pi$ network is most widely used in practical antenna tuning units [1-8] ).

For the $\pi$ network in figure 3 , the design specifications are given as $Q=10$ ( $Q$ is the circuit quality factor and can be used as a design parameter to represent the harmonic rejection performance [8] ), $R_{1}=50 \mathrm{ohm}, R_{2 \min }$ $=3 \mathrm{ohm}, \mathrm{R}_{2 \max }=2000 \mathrm{ohm}, f_{\min }=1.5 \mathrm{MHz}$, and $f_{\max }=$ $30 \mathrm{MHz}$. Using the method given in reference [6] , the component value ranges are determined as $\mathrm{C}_{1 \text { min }}=$ $273 \mathrm{pF}, \mathrm{C}_{1 \max }=0.0343 \mu \mathrm{F}, \mathrm{C}_{2 \min }=46.3 \mathrm{pF}, \mathrm{C}_{2 \max }=$ $0.136 \mu \mathrm{F}, \mathrm{L}_{\min }=0.0202 \mu \mathrm{H}$ and $\mathrm{L}_{\max }=13.9 \mu \mathrm{H}$. Note that $Z_{\text {in }}=j \omega C_{1} / /\left(j \omega L+j \omega C_{2} / / R_{2}\right)$ which can be used to calculate the VSWR. Tuning of the network for the different load resistance, $\mathrm{R}_{2}$ and frequency, $f$ using the method described has bee simulated. The results for $\mathrm{R}_{2}$ $=800 \mathrm{ohm}$ and $f=10.7 \mathrm{MHz}$ are presented in figures 4 .

From the results it can be seen that the proposed tuning algorithm works well with the matching task. It produces a high convergence rate. In particular, the graph in figure 4(a) indicates that the fitness of the bestfit citizen in the test will reach the acceptable VSWR value range of 1-1.5 within less than 10 generations. Furthermore, the final result gives approximately the ideal VSWR value. Figure 4(b) gives the values of fitness functions of certain generations. The data in figure $4(\mathrm{c})$ indicate the final component values and their respective binary codes which can be used to control switches in respective component arrays. The results obtained from this research are very encouraging and suggest that the proposed GA-based tuning method can be applied in the antenna tuning units with a highspeed microprocessor.

As shown in figure 1 the monitoring unit will supply data related to the input impedance $Z_{\text {in }}$ of the matching network after every tuning process is done by the control unit. The monitoring unit will then process these data to obtain the related VSWR, which will be the fitness of the tuned parameters. However, such a technique may not be feasible as the fitness of every produced set of parameters can only be obtained after tuning the matching network. Therefore, 500 times of tuning will be performed if a population of 10 is to be produced for a 50 generation cycle during the GA search process.

In order to overcome this problem, it can be proposed that the component ranges and all the related component values to be tirst entered into the program. The fitness of every parameter set produced will have to be determined through calculation and the matching network will only be tuned by using the final result.
However, this idea will not be able to guarantee the accuracy of the tuning process since the actual $\mathrm{Z}_{\mathrm{in}}$ value of the matching network is not being utilized to check every generated parameters set.

As for the matching network, difficulties in implementing the elements of small values for each component that is required to ensure high precision and the switching time needed will be expected. However, due to the availability of high component technology these difficulties can be overcome in hardware design.

\section{Conclusions}

A self-tuning method based on Genetic Algorithms for automatic impedance matching has been developed. The tuning strategies and algorithms have been discussed. The binary discrete arrangement of tunable components has been used. The VSWR has been used as an evaluation of fitness for each produced set of parameters. The tuning of $\pi$ impedance matching networks has been simulated using the proposed method. The simulated results have shown a very good performance and indicated that automatic impedance matching using Genetic Algorithms is feasible. The implementation of the tuning method using high-speed microprocessors and advanced component technology can further enhance the performance. Harmonic rejection information may also be directly incorporated into the proposed tuning process by modifying the fitness function. A whole microcomputer-based automatic tuning system using the tuning method given in the paper and $\pi$ matching network is now built up.

\section{References}

[1] P. Petrovic, M. Mileusnic and J. Todorovic. "Fast antenna tuners for high-power HF radio systems," ECCTD'89, IEE Conf. Pub. No.308, 1989

[2] P. A. Lewis and M. J. Underhill. "Quite tuning and matching of antennas for radiosilence Operation," IEE Proc. F, Vol.127, pp. 361-367, October 1980.

[3] J. Christensen. "Fast antenna tuners," Communication International, May, pp.22-27, 1987.

[4] M. J. Underhill and P. Lewis. "Automatic tuning of antennae," SERT Journal, Vol.8, pp.183-4, 1974.

[5] Yichuang Sun and J.K.Fidler. "Determination of impedance matching domain of passive LC ladder networks: theory and implementation, "Journal of The Franklin Institute. Vol.333(B), No.2, 1996

[6] Yichuang Sun and J.K.Fidler. "Component value ranges of tunable impedance matching networks in $R F$ communication systems," Proc. IEE Int. Conf. on Radio Frequency Systems and Techniques, UK, 1997.

[7] Yichuang Sun and J.K.Fidler. "High-speed automatic antemna tuning units," Proc IEE Int. Conf. 
Antennas and Propagation, No.407, pp. 218-222, Holland, 1995.

[8] Yichuang Sun and J.K.Fidler. "Design method for impedance matching networks," IEE Proc. Circuits Devices Systems, Vol.143, No.4, pp. 186-194, 1996.
[9] D. E. Goldberg. Genetic Algorithms in Search, Optimisation, and Machine Learning. Addison-Wesley Publishing Comp. Inc., pp.1-25 and 60-88, 1989.

[10] L. Davis, (ed). Handbook of Genetic Algorithms. Van Nostrand Reinhold, 1991.

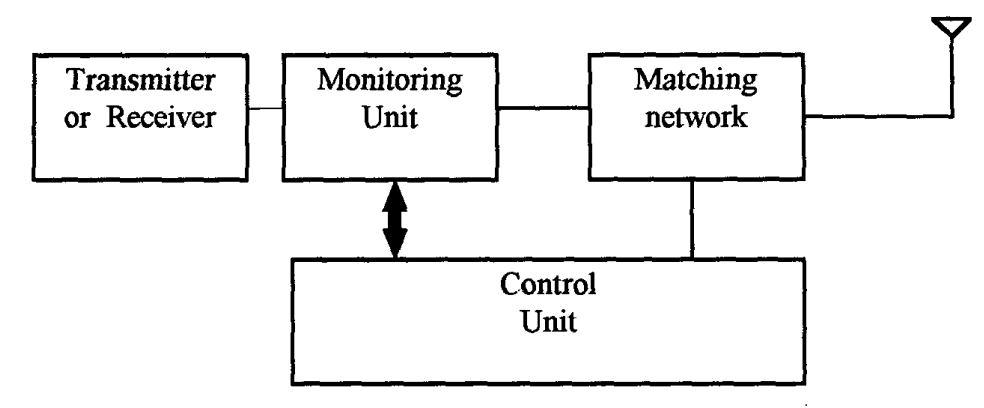

Figure 1 : Antenna Tuning Unit

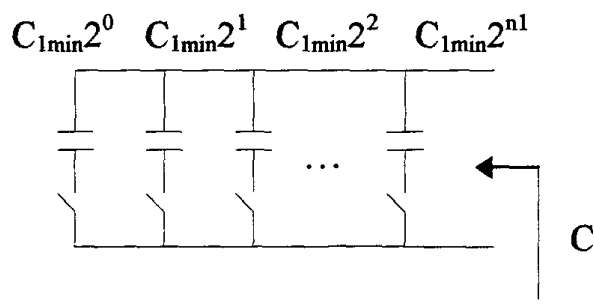

(a) Capacitor

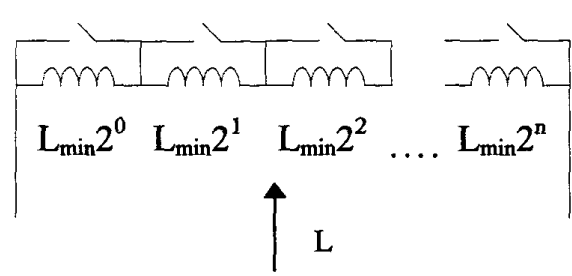

(b) inductor

Figure 2 : Binary Tuning Array Arrangement

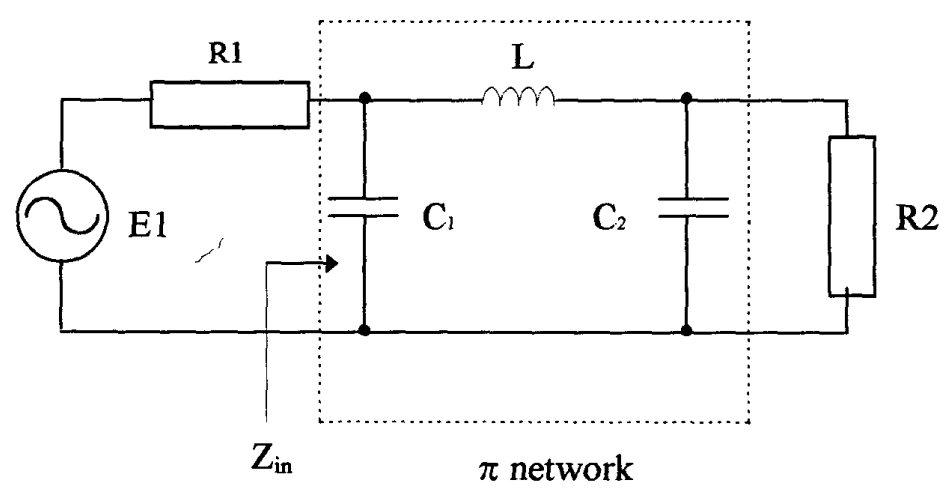

Figure 3: Impedance Matching Network 


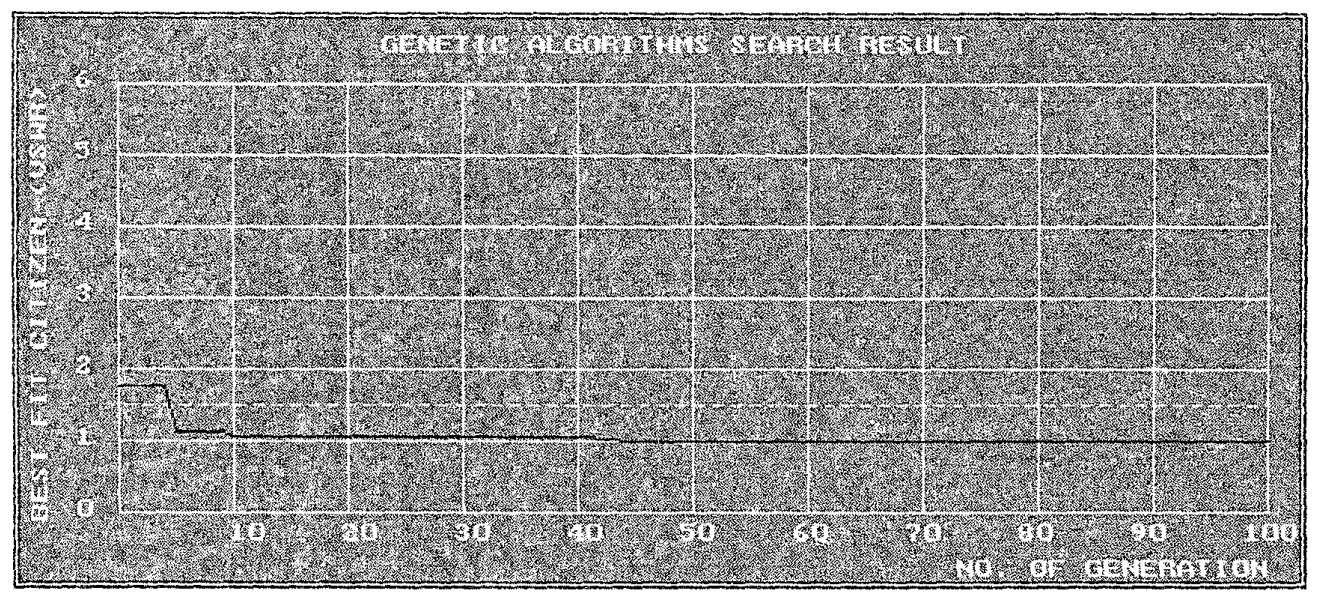

(a)

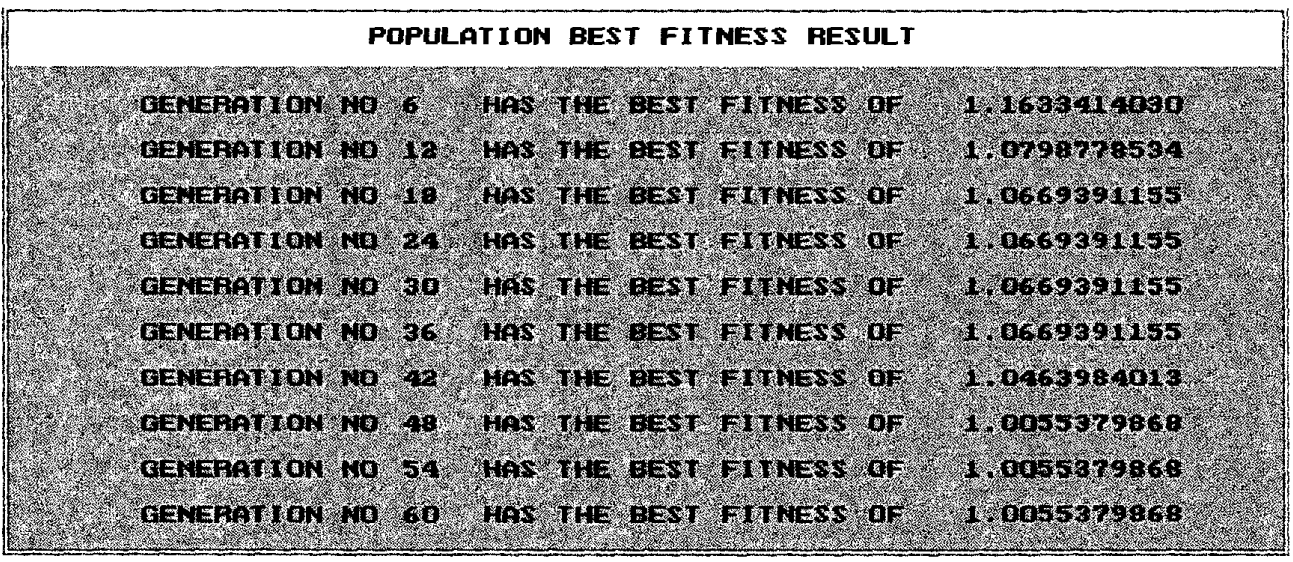

(b)

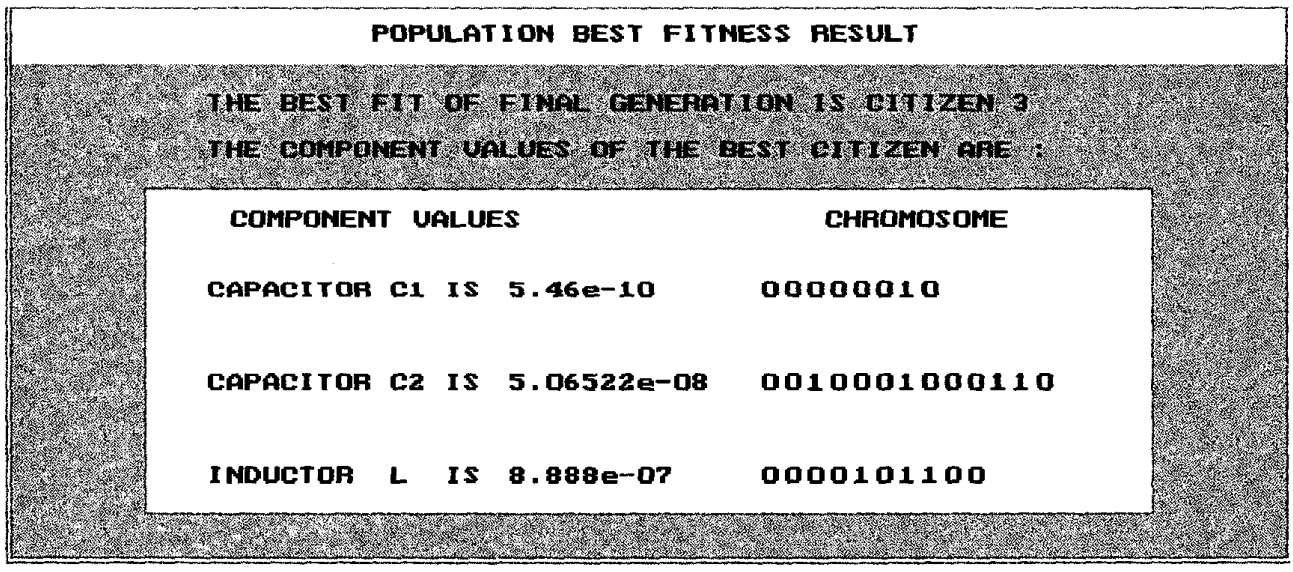

(c)

Figure 4 Simulated results 